\title{
Los usos de las TIC en la mercantilización de la universidad: análisis de los documentos oficiales del EEES
}

\author{
Begoña BALlesteros CARRAsco \\ Universidad Complutense de Madrid \\ begoballesteros@ccinf.ucm.es \\ Daniel Franco Romo \\ Universidad Complutense de Madrid \\ dfrancoromo@ccinf.ucm.es \\ Joan PEDRo CARAÑANA \\ Universidad Complutense de Madrid \\ joan.pedro@pdi.ucm.es
}

Recibido: 20/07/2012

Aceptado: 09/10/2012

Resumen: La construcción del Espacio Europeo de Educación Superior (EEES) está transformando profundamente la universidad. Los cambios en curso orientan a la institución universitaria hacia un modelo en el que la rentabilidad económica y el aprovechamiento empresarial del conocimiento pasan a detentar el mayor protagonismo. Las Tecnologías de la Información y de la Comunicación (TIC) juegan un papel estratégico en este proceso. Mediante el análisis de los documentos en los que se define el proyecto del EEES, el artículo aborda los usos de las TIC que se están promoviendo desde las instituciones oficiales para contribuir a la actual transformación de la universidad.

Palabras clave: capitalismo, mercantilización, TIC, universidad.

\section{The uses of ICT in the commodification of the university: An analysis of the EHEA official documents}

\begin{abstract}
The construction of the European Higher Education Area (EHEA) is profoundly transforming the university. Changes underway orient the university institution toward a model in which economic profitability and private utilization of knowledge wield a greater role. Information and Communication Technologies (ICT) play a strategic role in this process. By analyzing the documents that define the project of the EHEA, the article discusses the uses of ICT being promoted by official institutions to contribute to the ongoing transformation of the university.
\end{abstract}

Keywords: capitalism, commodification, ICT, university

\section{Referencia normalizada}

BALLESTEROS CARRASCO, Begoña; FRANCO ROMO, Daniel y PEDRO CARAÑANA, Joan (2012): "Los usos de las TIC en la mercantilización de la universidad: análisis de los documentos oficiales del EEES". Estudios sobre el mensaje periodístico. Vol. 18, núm. especial octubre, págs.: 101-110. Madrid, Servicio de Publicaciones de la Universidad Complutense.

Sumario: 1. Introducción. 2. Metodología. 3. Desarrollo; 3.1. Los fines que se persiguen con la transformación de las universidades europeas; 3.2. Los usos de las TIC que se proponen para la transformación de la universidad y la consolidación de la Europa del conocimiento. 4. Conclusiones. 5. Referencias bibliográficas. 


\section{Introducción}

El proceso de construcción del Espacio Europeo de Educación Superior (EEES) trae consigo profundas transformaciones que afectan a la organización de la universidad en su conjunto, a su funcionamiento y, en consecuencia, a la misión que la sociedad le encomienda en cada momento histórico. La denominada "universidad de masas", que fue construyéndose bajo el modelo de acumulación capitalista surgido de la segunda guerra mundial, está siendo sustituida -primero paulatinamente y ahora a marchas forzadas en el contexto de la crisis actual- por otra en la que la rentabilidad económica de las enseñanzas universitarias, la comercialización de los resultados de la actividad investigadora y, en general, el aprovechamiento empresarial del conocimiento pasan a detentar un protagonismo hasta ahora desconocido.

Las instituciones europeas, encargadas de definir las directrices de este proceso, hacen hincapié en la necesidad de adecuar la universidad a las demandas de la sociedad actual, concretamente, a la llamada "sociedad del conocimiento". A pesar de las connotaciones positivas del término, numerosos análisis se han encargado de desmitificar la idea de "sociedad del conocimiento", indicando que "no tiene nada que ver con una sociedad de ciudadanos sabios", sino más bien "con la constatación de que el aumento de la productividad y la competitividad pasa, cada vez más, por la innovación, que queda definida no como la producción de conocimientos nuevos, sino como su difusión económicamente rentable" (Alegre Zahonero y Fernández Liria, 2004: 235).

En este contexto, no resulta extraño que la mercantilización de la universidad constituya uno de los tópicos más frecuentes entre quienes se están preocupando en España de analizar la actual reforma universitaria desde posiciones críticas (vid., por ejemplo, Fernández Liria y Serrano García, 2009; Ferreiro Baamonde, 2010; Fuentes Ortega, 2005 y 2007; Galcerán Huguet, 2003 y 2010; Sevilla Alonso, 2010). La mercantilización de la enseñanza superior también está siendo discutida en otros países, lo que sugiere que no se trata de un fenómeno meramente español, ni siquiera europeo, sino sistémico (vid., por ejemplo, Bailey y Freedman, 2011; Scott, 2012; Wallerstein, 2012). Estos análisis coinciden en percibir las transformaciones en curso como una reconversión de las universidades en empresas al servicio del mercado. Reconversión que compromete, entre otras cosas, el carácter público y universal de la educación, la autonomía universitaria, el pensamiento crítico y la producción desinteresada de conocimientos. Como resultado, la institución universitaria se desvincula cada vez más de la reflexión científica, social, humanística y ética, para priorizar los saberes que generen beneficios económicos.

Siguiendo esta línea de trabajos que han advertido de la instrumentalización económica de la educación superior, este artículo trata de corroborar esa dinámica mediante el análisis de los usos de las Tecnologías de la Información y de la Comunicación (TIC) que se están promoviendo para contribuir a la adaptación de la universidad a los requisitos del EEES. Constituye una idea constantemente repetida que las TIC juegan un papel estratégico en este proceso. La penetración e influencia de grandes empresas tecnológicas en la universidad, la formación en competencias en el uso de TIC que son demandadas por un mercado laboral en permanente muta- 
ción o la creación de empresas de base tecnológica son tópicos comunes en la literatura sobre el EEES.

El objeto de este trabajo consiste en identificar qué usos concretos de las TIC se están promoviendo desde los organismos oficiales y a qué cambios se vinculan dichos usos, en la universidad en particular y en la sociedad en su conjunto. Para ello, se han analizado una serie de documentos en los que se recogen las líneas fundamentales para el diseño y la materialización del EEES. Los resultados de este análisis se han interpretado en clave sociohistórica; es decir, relacionando los usos de las TIC que se vinculan al cambio en la universidad con uno de los rasgos definitorios de la Europa actual: su carácter de sociedad capitalista monopólica y globalizada, con dificultades crecientes y estructurales para obtener rentabilidad. Se confirmará así que el empleo de las TIC responde a la misma lógica mercantil que caracteriza a la transformación en curso del sistema de enseñanza superior en su conjunto. Entendemos que un análisis de este tipo puede ayudar a comprender críticamente qué proyecto político subyace en los cambios que está viviendo la institución universitaria, así como a captar el sentido histórico de tales transformaciones.

\section{Metodología}

Se ha realizado un análisis de contenido de una serie de documentos oficiales en los que se define el proyecto del EEES. En concreto, se han analizado diecisiete textos, entre los que se encuentran:

- Documentos en los que se sientan las bases para la armonización de la educación superior europea: la "Magna Charta Universitatum" (1988) -primer antecedente explícito del proyecto de convergencia de los sistemas universitarios-, la "Declaración de la Sorbona" (1998), la "Declaración de Bolonia" (1999) o la "Declaración de Praga" (2001); las comunicaciones de la Comisión Europea "El papel de las universidades en la Europa del conocimiento" (2003), "Movilizar el capital intelectual de Europa: crear las condiciones necesarias para que las universidades puedan contribuir plenamente a la estrategia de Lisboa" (2003), "Cumplir la agenda de modernización para las universidades: educación, investigación e innovación" (2006), "Una Agenda Digital para Europa" (2010); y la decisión del Consejo y del Parlamento europeos "por la que se establece un programa de acción en el ámbito del aprendizaje permanente" (2006).

- Documentos sobre la implantación del EEES en España: el "Informe Universidad 2000" (2000), el documento-marco "La integración del sistema universitario español en el Espacio Europeo de Enseñanza Superior" (2003), la "Ley Orgánica 4/2007, de 12 de abril, por la que se modifica la Ley Orgánica 6/2001 de 21 de diciembre, de Universidades" (2007), la "Estrategia Universidad 2015" (2010) y el informe "Audacia para llegar lejos: universidades fuertes para la España del mañana” (2011). 
- Documentos en los que se incide en la importancia de incorporar y desarrollar el uso de las TIC en las enseñanzas universitarias: el informe de la Comisión Europea "Concebir la educación del futuro: promover la innovación con las nuevas tecnologías" (2000), la decisión del Consejo y el Parlamentos europeos "por la que se adopta un programa plurianual (2004-2006) para la integración efectiva de las tecnologías de la información y la comunicación (TIC) en los sistemas de educación y formación en Europa (programa eLearning)" (2003) y el informe final del proyecto Tuning (2003).

El procedimiento del análisis ha consistido en identificar, de forma sistematizada:

- Primero, de qué manera las instituciones rectoras de este proceso vinculan los usos de las TIC con los cambios que se están llevando a cabo en la universidad para la construcción del EEES.

- Y, segundo, cómo esa transformación de la universidad, facilitada por las TIC, se pone en relación en los documentos oficiales con el nuevo escenario social, macroeconómico y laboral que se va conformando en Europa tras la crisis de los años setenta del siglo XX.

Se ha trabajado, así, con un modelo de análisis que trata de captar las relaciones entre: capacidades de las TIC [para facilitar el] cambio en la universidad [contribuyendo con ello a la] transformación de la sociedad.

Los resultados obtenidos se sintetizan en el siguiente apartado.

\section{Desarrollo}

\subsection{Los fines que se persiguen con la transformación de las universidades euro- peas}

En los documentos analizados, la nueva universidad que se pretende construir no se propone en abstracto, como un fin en sí mismo, sino vinculada a cambios de más largo alcance orientados a consolidar un nuevo escenario social denominado, genéricamente, "Europa del conocimiento". El vínculo de la nueva universidad con ese escenario es un denominador común al conjunto de textos revisados, ya se mencione explícitamente o quede referido de manera implícita. En concreto, se señalan dos tipos de fines a los que la universidad puede y debe contribuir:

- La consolidación de los avances sociopolíticos que han distinguido a Europa desde el fin de la segunda guerra mundial. La institución universitaria puede contribuir a lograr, conservar o mejorar aspectos como la cohesión social, la igualdad de oportunidades, el bienestar de las personas, el respeto por los derechos humanos, la tolerancia hacia otros pueblos y culturas, la protección del medio ambiente en beneficio de las generaciones futuras, las libertades políticas o la realización personal de los ciudadanos europeos. 
- El desarrollo de una economía basada en el conocimiento. Se trata, según se desprende de los documentos analizados, del tipo de economía que permitirá un crecimiento sostenible y que contribuirá a crear más y mejores puestos de trabajo. Las universidades constituyen un pilar fundamental para la consecución de este objetivo, como generadoras de conocimiento y como formadoras de las nuevas generaciones de trabajadores en aquellos saberes y habilidades que les faciliten su inserción en el mundo laboral y su reciclaje a lo largo de su trayectoria profesional.

Para conseguir estos fines, los documentos analizados sostienen que resulta ineludible una profunda transformación de la universidad. Y, para ello, las TIC deben ser aprovechadas, en algunos de esos cambios y en el sentido que se muestra a continuación.

\subsection{Los usos de las TIC que se proponen para la transformación de la universi- dad y la consolidación de la Europa del conocimiento}

Como en tantos otros sectores, las TIC son concebidas por las instituciones oficiales como un elemento necesario -aunque no sea suficiente- en las transformaciones que deben acometerse en la educación superior para la construcción del EEES. A través del análisis de los documentos seleccionados, se han identificado siete ámbitos susceptibles de cambio en la universidad en los que las tecnologías digitales están implicadas de manera estratégica:

1) Eficiencia en el funcionamiento administrativo. Como se sabe, la digitalización permite racionalizar y depurar el modelo de gestión de los recursos materiales y humanos de una organización. Con la ayuda de estos avances tecnológicos, los centros de enseñanza superior han de convertirse en instituciones con burocracias ágiles.

2) Calidad y competitividad en el servicio. La globalización de la enseñanza superior obliga a las universidades europeas a competir entre sí y con otras universidades no europeas para atraer financiación, nuevos estudiantes y los mejores profesores e investigadores. Deben convertirse, por tanto, en centros de referencia internacional, lo que supone ofrecer, entre otras cosas, un entorno tecnológico puntero (redes, equipamiento para la investigación y el estudio, servicios web y de correo, digitalización de fondos, etc.).

3) Internacionalización y movilidad. Las universidades han de abrirse las unas a las otras creando lazos y sinergias entre distintos centros, dentro y fuera de la UE y con otros sectores no universitarios. Las herramientas digitales permiten, en este sentido, establecer relaciones académicas sin las restricciones de distancia y tiempo que había en otras épocas. Facilitan, con ello, la puesta en marcha de proyectos formativos y/o de investigación conjuntos por parte de centros alejados geográficamente entre sí. 
4) Explotación comercial de resultados científicos. A la tradicional labor de los centros universitarios de generar conocimiento nuevo, se le añade ahora la tarea de convertir los avances científicos y tecnológicos generados en la investigación en resultados tangibles y comercializables. Las TIC deben ayudar a que la universidad se convierta en ese nexo entre el desarrollo del conocimiento y el desarrollo económico: facilitan los vínculos entre el mundo universitario y los sectores empresariales; favorecen la creación de empresas (spin-off) con alto contenido tecnológico y menor coste; y fomentan una actitud emprendedora entre quienes han sido formados y capacitados con pedagogías y contenidos innovadores.

5) Organización flexible e interdisciplinar de la actividad investigadora. Las TIC están alterando la organización de los procesos de trabajo en general y, con ello, también la del trabajo de investigación que se lleva a cabo en las universidades. Con la ayuda de las tecnologías digitales, los grupos de investigación pueden adoptar formas organizativas más flexibles e interdisciplinares, que les permitan descubrir y explotar nuevos campos de innovación, y amoldarse, en cada momento, a las demandas que les lleguen de la sociedad y de su tejido empresarial.

6) Aprendizaje permanente. Las universidades deben aumentar su oferta de formación continua, con el objetivo de facilitar el reciclaje constante de trabajadores que sólo pueden estudiar a tiempo parcial, desde su casa o desde su lugar de trabajo. Las TIC permiten ofrecer educación a distancia, mediante soportes como los campus virtuales, el correo electrónico u otras plataformas multimedia.

7) Digitalización del proceso de enseñanza. La universidad debe poner todos los medios a su alcance para ofrecer una enseñanza "innovadora y digital". La UE promueve en este sentido el desarrollo de planes de estudios duales, que combinen aprendizaje tradicional y en línea, y un nuevo modelo de enseñanza centrado en el alumno, que deberá adquirir nuevas competencias y habilidades prácticas relacionadas con las TIC, en el entendimiento de que así se facilitará su inserción en el mercado laboral.

\section{Conclusiones}

El análisis realizado pone de manifiesto que los usos de las TIC y la transformación de la universidad que se promueven desde los documentos oficiales están atravesados por un objetivo común: consolidar la penetración de la lógica mercantil en la estructura de la institución universitaria. Así cabe entender, por ejemplo, la concepción competitiva del funcionamiento de la universidad, que debe luchar con otras universidad para atraer financiación, investigadores de renombre y nuevos estudiantes; el aprovechamiento comercial del conocimiento científico; o la reorientación de la oferta formativa hacia contenidos prácticos y constantemente actualizados que pueda satisfacer las exigencias que en cada momento tenga el mercado de trabajo. Como avanzó Bauman:

"Con la revolución tecnológica permanente que convierte un valor como los conocimientos y hábitos adquiridos en un hándicap, con la utilidad cada vez 
más breve que tienen las habilidades prácticas, cuya vigencia desaparece antes de lo que se tarda en adquirirlas y certificarlas con un título universitario, la formación profesional $a d-h o c$ y a corto plazo [...] se vuelve más atractiva (y, de hecho, es una opción más razonable) que la educación universitaria de pleno derecho que ya no es capaz de asegurar una carrera profesional de por vida" (Bauman, 1997: 49).

Esta reorganización de la institución universitaria, materializada en el proyecto del EEES, debe entenderse desde una perspectiva temporal amplia. Esto es, como algo enraizado en el devenir histórico de las sociedades capitalistas y, en concreto, en los cambios derivados del paso del capitalismo industrial al monopólico-globalizado de nuestros días. En esta nueva etapa, las sociedades capitalistas están atravesadas, en su conjunto, por una crisis estructural y permanente arrastrada desde los años setenta. Crisis que está originada, fundamentalmente, por las dificultades crecientes para obtener rentabilidad de las inversiones (Gill, [1996] 2002: 540-567) ${ }^{1}$.

Ante dichas dificultades, el sistema capitalista necesita encontrar nuevas formas de valorización y, para ello, va insertando paulatinamente en el circuito de acumulación sectores que permanecían al margen de los mecanismos del mercado, como sucedía con buena parte de las estructuras y de los organismos de la educación superior. Hasta ese momento, se entendía que el avance y la transmisión de los saberes era algo que tenía que estar razonablemente separado de la lógica mercantil y de las necesidades económicas inmediatas. Pero, a partir de los años setenta, las élites económicas y políticas empiezan a señalar la necesidad de reacomodar la universidad a las nuevas condiciones históricas y, en consecuencia, a concebirla como una oportunidad de negocio. De ahí que los usos de las TIC que se han analizado puedan interpretarse como parte de esa adaptación de la universidad al nuevo contexto macroeconómico, cada vez más necesitado de rentabilidad. Esta necesidad implica la supeditación creciente de la institución universitaria al logro de la máxima ganancia con la mayor inmediatez posible y al margen de los costes sociales que este giro pueda comportar. Se trata, por tanto, de unos usos de las TIC esencialmente instrumentales, orientados a transformar una universidad que también está siendo instrumentalizada para cumplir con las necesidades económicas de las sociedades capitalistas globalizadas.

Pero, al mismo tiempo, esos usos se revelan, a nuestro entender, incompatibles con la mejora de las condiciones de vida del conjunto de la población, objetivo que atraviesa -de manera más o menos explícita- los documentos analizados. La cohesión so-

1 Como han mostrado numerosos estudios económicos (vid., por ejemplo, Álvarez Peralta, 2012), la actual crisis, de origen financiero, que afecta a Europa y a otros países desarrollados desde 2007 es reflejo, precisamente, de esas dificultades crecientes para obtener un suficiente nivel de rentabilidad. Desde los años ochenta, el capital ha tenido que recurrir al circuito especulativo para revalorizarse de manera rápida, con los costes sociales que ahora se están manifestando con toda su intensidad. No es casualidad que el estallido de esta crisis haya sido instrumentalizado para reducir la financiación pública de la educación superior y dar un impulso al nuevo modelo de universidad. 
cial, la sostenibilidad medioambiental, la igualdad de oportunidades o el desarrollo democrático y social -aspectos todos ellos mencionados en los textos revisados como justificación de los cambios que se pretenden implantar en la universidad-, encuentran difícil acomodo en una institución orientada, principalmente, a la obtención de ganancia económica inmediata. Cuando las instituciones oficiales vinculan en sus documentos la subordinación de la universidad a la lógica de la rentabilidad con el progreso de la sociedad en su conjunto, están omitiendo que, como se sabe, bajo formas de producción capitalistas, no es posible la conciliación entre la racionalidad instrumental que orienta los procesos productivos y la racionalidad liberadora que busca la emancipación del ser humano y debería orientar, en último término, la gestión y funcionamiento de las universidades.

Tal y como se desprende del análisis realizado, el proyecto del EEES parece encaminado a que la racionalidad instrumental se imponga a la racionalidad liberadora en la concepción de la educación superior, con todo lo que esto puede implicar, a medio y largo plazo, en la generación de conocimiento y en la formación de las nuevas generaciones. La actual reorganización de la universidad tiene ya el suficiente camino recorrido como para poder afirmar que no supone un simple cambio en su modelo de gestión. Se está produciendo, al mismo tiempo, una transformación en las funciones que la sociedad le ha atribuido históricamente: de ser la principal institución generadora, depositaria y transmisora del saber puede quedar reducida a un espacio para la enseñanza de habilidades instrumentales y la producción de conocimiento orientado a las necesidades del mercado.

\section{Referencias bibliográficas}

ALEGRE ZAHONERO, Luis y FERNÁNDEZ LIRIA, Carlos (2004): "La revolución educativa. El reto de la Universidad ante la sociedad del conocimiento". Logos. Anales del Seminario de Metafisica, vol. 37. Madrid, Universidad Complutense de Madrid, pp. 225-253.

ÁLVAREZ PERALTA, Ignacio (2012): "Capital financiero, Estado y crisis financiera en Europa". Encrucijadas. Revista Crítica de Ciencias Sociales, $\mathrm{n}^{\circ} 3$. Salamanca, Asociación Contubernio, pp. 7-14.

BAILEY, Michael y FREEDMAN, Des (eds., 2011): The Assault on Universities. A Manifesto for Resistance. London, Pluto Press.

BAUMAN, Zygmunt (1997): “The Present Crisis of the Universities", en BRZEZINSKI, Jerzy y NOWAK, Lersek (eds.): The Idea of the University. Amsterdam y Atlanta, Rodopi B.V., pp. 47-54.

FERNÁNDEZ LIRIA, Carlos y SERRANO GARCÍA, Clara (2009): El plan Bolonia. Madrid, Catarata.

FERREIRO BAAMONDE, Xulio (2010): "Mercantilización y precarización del conocimiento: el proceso de Bolonia", en VV.AA.: La Universidad en conflicto. Capturas y fugas en el mercado global del saber. Madrid, Traficantes de Sueños, pp. 113-142. 
FUENTES ORTEGA, Juan Bautista (2005): "El espacio europeo de educación superior, o la siniestra necesidad del caos". Logos. Anales del Seminario de Metafísica, vol. 38. Madrid, Universidad Complutense de Madrid, pp. 303-335 .

FUENTES ORTEGA, Juan Bautista (2007): "Para una crítica de la idea de «flexibilidad profesional». La relación entre la historia de la psicología y de las ciencias humanas y los saberes humanísticos". Revista de Historia de la Psicología, vol. 28, $\mathrm{n}^{\circ} 1$. Valencia, Universidad de Valencia, pp. 19-42.

GALCERÁN HUGUET, Montserrat (2003): "El discurso oficial sobre la Universidad". Logos. Anales del seminario de metafísica, $\mathrm{n}^{\circ}$ 36. Madrid, Universidad Complutense de Madrid, pp. 11-32.

GALCERÁN HUGUET, Montserrat (2010): "La mercantilización de la universidad". Revista Electrónica Interuniversitaria de Formación del Profesorado, n ${ }^{\circ} 32$ (13, 2). Zaragoza, Asociación Universitaria de Formación del Profesorado, pp. 89-106: http://www.aufop.com/aufop/revistas/indice/digital/147. [fecha de consulta: 14 de septiembre de 2012].

GILL, Louis [1996] (2002): Fundamentos y límites del capitalismo. Madrid, Trotta.

SCOTT, Debra Leigh (2012): "How the American University was Killed, in Five Easy Steps", en The Homeless Adjunct: http://junctrebellion.wordpress.com/2012/08/12/howthe-american-university-was-killed-in-five-easy-steps/. [fecha de consulta: 14 de septiembre de 2012].

SEVILLA ALONSO, Carlos (2010): La fábrica del conocimiento. La Universidadempresa en la producción flexible. Madrid, El Viejo Topo.

WALLERSTEIN, Immanuel (2012): "Higher Education Under Attack", Commentary No. 324, en Commentaries - Fernand Braudel Center - Binghamton University: $\mathrm{http}: / / \mathrm{www} 2$. binghamton.edu/fbc/commentaries/archive-2012/324en.htm. [fecha de consulta: 14 de septiembre de 2012]. 


\section{Begoña BALLESTEROS CARRASCO}

begoballesteros@ccinf.ucm.es

Universidad Complutense de Madrid

Doctora Europea en Comunicación, Cambio Social y Desarrollo por la Universidad Complutense de Madrid. Miembro del Grupo de Investigación UCM Identidades Sociales y Comunicación.

\section{Daniel FRANCO ROMO}

dfrancoromo@ccinf.ucm.es

Universidad Complutense de Madrid

Doctor Europeo en Comunicación, Cambio Social y Desarrollo por la Universidad Complutense de Madrid. Miembro del Grupo de Investigación UCM Identidades Sociales y Comunicación.

\section{Joan PEDRO CARAÑANA}

joan.pedro@pdi.ucm.es

Universidad Complutense de Madrid

Doctorando en el programa Comunicación, Cambio Social y Desarrollo de la Universidad Complutense de Madrid. Miembro del Grupo de Investigación UCM Identidades Sociales y Comunicación. 